\title{
How diabetic patients think about and manage their illness in Taiwan
}

\author{
W. A. Lai, C.-Y. Lew-Ting* and W.-C. Chiet
}

Department of Family Medicine, Taichung Veterans' General Hospital, Taichung, *Department of Public Health and Graduate Institute of Health Policy and Management, College of Public Health, National Taiwan University, Taipei and TDepartment of Public Health and Graduate Institute of Preventive Medicine, College of Public Health, National Taiwan University, Taipei, Taiwan

Accepted 16 March 2004

\begin{abstract}
Aims Type 2 diabetes is becoming more prevalent in Taiwan. Growing evidence suggests a patient-centred approach is more effective in facilitating self-management than a professional-centred one. The aim of this qualitative study was to investigate Chinese diabetic patients' perceptions about their illness and treatment strategies to facilitate patient-centred, culture-sensitive clinical skills.
\end{abstract}

Methods We used in-depth interviews to collect perspectives from 22 participants recruited from a rural Taiwan community. All interviews were audio-taped and the transcripts were analysed by editing and immersion/crystallization. Emerging themes were compared with current medical knowledge to determine their clinical significance.

Results Generally, Chinese diabetic patients had variable perceptions about different treatment strategies. All agreed that dietary restriction and exercise were beneficial. The former, mainly understood as reducing carbohydrate intake, was thought to be most important; exercise, to the point of sweating, was seen as a way to eliminate pharmaceutical toxins. Taking medicine was regarded with ambivalent attitudes due to concerns about adverse effects (especially renal injury).

Conclusions Patients regarded all treatment strategies as integrative and intertwined in daily life. There are three misconceptions which we found should be clarified during medical consultations: (i) focusing on sugar-control only; (ii) benefit of sweating to eliminate toxins; (iii) fear of renal toxicity of hypoglycaemic agents. The renaming of diabetes, to such as 'metabolic syndrome', may bring new, acceptable insight to Chinese diabetic patients.

Diabet. Med. 22, 286-292 (2005)

Keywords diabetes mellitus, illness perceptions, in-depth interview, self-care strategy, Taiwan

\section{Introduction}

Type 2 diabetes mellitus (DM) and its complications are becoming more prevalent in Taiwan [1] as in other areas of the world [2]. Since several clinical trials have demonstrated benefits of aggressive treatment $[3,4]$, which rely heavily on selfmanagement [5-7], the development of more effective behavioural strategies to achieve the maximum benefit is needed.

To gain a better understanding of human health behaviour, many researchers have examined cognitive factors such as

Correspondence to: Wei-Chu Chie, Room 209, 19 Hsuchow Road, Taipei, 10020, Taiwan. E-mail: weichu@episerv.cph.ntu.edu.tw attitudes to health care, perceptions and knowledge about diseases [8-10], which are open to change and enable the development of more effective behavioural interventions [11]. Among the developed models involving cognitive factors, the health belief model (HBM) is widely used [11], but suffers from focusing only on factors in a motivational phase and neglecting the volitional phase where action is planned, performed and maintained $[12,13]$. It leads to a narrow conception of culture and human action $[9,14]$.

Kleinman [15], has proposed a context-rich, culturesensitive explanatory model (EM) with five components including aetiology, time and mode of onset of symptoms, patho-physiology, course of sickness, and treatment. Diefenbach 
and Leventhal [13] proposed a similar construct 'illness representation', which also has five attributes including identity, timeline, causal, controllability and consequences. These personal illness models provide a dynamic framework addressing the changing nature of the lay illness beliefs $[13,15]$. Hampson and colleagues [16], drawing on both perspectives, have adopted a model with broader definition, and demonstrated its applicability $[17,18]$.

With an understanding that different perceptions between patients and physicians are an important cause of conflict or patient's non-adherence $[15,19]$, some clinical experts have proposed a new patient-centred clinical method [20-23]. It recommends more attention to patients' expectations, thoughts, feelings, ways he/she experiences illness and seeing them as collaborators $[21,22]$. Investigating a patients' personal model may facilitate more effective patient-physician relationships, obtain more thorough historical data and then increase patients' adherence [20,23].

Previous work exploring diabetic patient's perceptions about illness has generally been undertaken in Europe or North America [10,16-18,24-29]. Chinese culture with its unique medical tradition has different perspectives on disease [30], medicine [31] and behavioural approaches to chronic disorders [32,33].

In general, Chinese medicine, based on ancient Chinese philosophy, highlights a holistic approach towards health, which was believed to be a state of spiritual and physical harmony with nature. It recognizes that the body has more than 12 meridians. Each meridian, governed by one organ, represents its health state. The physicians are expected to look for the underlying yin-yang imbalances of each meridian, discriminate pattern and find its underlying cause. They combine the use of medicinal herbs, acupuncture, dietary therapy, massage and therapeutic exercise to modulate the flow of energy (qi) through all meridians to cure disorders or promote health [32,33].

In Jayne and Rankin's work conducted in a US west coast Chinatown health centre [34] the authors argued that, in addition to alien environmental stressors, Chinese diabetic immigrants faced more challenges in understanding, coping and managing their illness. Language was a major barrier. In their pioneering study, however, few themes extracted were based on Chinese cultural viewpoints.
The aim of this study was to describe Chinese Type 2 diabetic patients' perceptions of their illnesses and self-care strategies. We used in-depth interviews to collect their first-hand perspectives with an interview protocol based on synthesizing models suggested by Kleinman [15], Diefenbach and Leventhal [13], and Hampson [16]. We hoped our results might help clinicians to develop more patient-centred, culture-sensitive clinical skills.

\section{Patients and methods}

\section{Patients}

Our study was performed in a rural town in central Taiwan. This town, with a population of around 70000 , has several economic activities, including agriculture, fishing and light industry. People in this town, typical of those in Taiwan, were immigrants or offspring of immigrants who had come from mainland China decades to hundreds years ago. Traditional Chinese medicine (TCM) is still popular and supported by government policy as it is reimbursed by National Health insurance.

We adopted a sampling strategy of maximum-variation, which was designed to document diverse variations and identify important common patterns [35]. We tried to enroll patients with different demographic backgrounds to avoid omitting some important patterns of layman perspectives. With the help of public health nurses, we recruited 22 diabetic patients with a duration of illness more than one year. Of them, 12 were males, the average age was 60.2 years old (between 44 and 80 ) and the average duration of illness was 8.3 years (between 2 and 25 years) (Table 1 ).

\section{Data collection and analysis}

This manuscript is part of the $\mathrm{PhD}$ dissertation of $\mathrm{Dr}$ Lai (the first author). The study was approved by the $\mathrm{PhD}$ dissertation advisory committee and the Ethical Committee of College of Public Health, National Taiwan University. The first author interviewed the 22 participants in depth and audio-taped the dialogue with the informed verbal consent of each participant. All the names mentioned in text are pseudonyms and do not refer to any specific participant. To make participants feel comfortable talking, the interviews were conducted in the health station or other familiar community settings. Oral consent witnessed by the first author (interviewer) was obtained from each patient
Table 1 Patient socio-demographic characteristics and diabetes-related health status

\begin{tabular}{ll}
\hline Characteristic & Number or mean (range) \\
\hline Gender: male/female & $12 / 10$ \\
Mean age (range), years old & $60.2(44-80)$ \\
Mean length with diabetes (range), years & $8.3(2-25)$ \\
Occupation or previous occupation (retired): & \\
farmer/businessman/labourer/officer/soldier/teacher/housekeeper & $3 / 2 / 5 / 1 / 1 / 2 / 8$ \\
Status of diabetes (HgbA $1 \mathrm{c}$ level): <7/7-9/> 9/unknown & $9 / 4 / 2 / 7$ \\
Co-morbidity: HT/(PDR + BDR)/smoking/CV disorder $\dagger$ & $9 /(2+1) / 3 / 3$ \\
Family history of diabetes mellitus & 9
\end{tabular}

†BDR, background diabetic retinopathy; CV, cardiovascular; HT, hypertension; PDR, proliferative diabetic retinopathy. 
after explaining the aims and methods of this study and promising confidentiality.

The interview protocol included three parts of questions: (i) illness experiences since diagnosis; (ii) viewpoint on diabetes including cognitive and emotional aspects; (iii) the contextual factors related to diabetic and health conditions. Furthermore, to highlight clinical applications in the present study, we paid greater attention to participants' perceptions of the major treatment strategies (including diet control, exercise and pharmaceuticals).

At the first stage of data analysis, we combined strategies of editing and immersion/crystallization styles. The former searches for meaningful segments, consisting of cutting, pasting and rearranging until a reduced meaningful summary emerges. The latter is undertaken by the analyst's prolonged immersion into and experience of the text and emerging with an intuitive crystallization [35].

Finally, we compared the emerging themes with current recommendations of the American Diabetes Association [3638] and the third report of the National Cholesterol Education Program-Adult Treatment Panel III (NCEP ATP-III) [39]. All the authors read the verbatim, participated in discussion and reached a consensus on emerging themes and edited results.

\section{Results}

We focus our report on the rationale and behaviour of our participants in relation to three domains of self-care management: dietary management, exercise practice, and pharmaceutical treatment.

\section{Dietary management}

\section{Importance of cutting sugary food}

All of our participants, even those refusing 'scientific' medicines, agreed that dietary management is beneficial. Miss Lai, a middle-aged white-collar employee said:

'... If I tell my mom to take medicine, my first younger brother, a yi-guan-dao (a Chinese traditional religion) believer, will say "don't take drugs, just use food remedy, ... it'll get better by eating stuff, eating vegetarian food" ...'

It was believed that food is more compatible with natural life than artificial drugs. Mr Chen's comments were typical of our patients:

'It's never good to take too much (medicine). I'm afraid the compound, western medicines, may ...'

To most participants (16/22) in our study, dietary management was referred to as mainly to cutting down sweets or carbohydrates. Some of them even said that dietary management was more important than drugs. Mr Ho said:

'You may start off with a low dosage, but as the years go by, even though you take more and more drugs, they don't have any effect. Your "sugar drugs" are just like that. The way that I see it, self-control is more important ..., not eating too much rice and trying to cut down on sugar,... that's all, nothing else, nothing ... (How about taking medicine?) Forget it; if you don't control your diet, taking medicine won't do any good.'

\section{Practical limitation in daily life}

These patients also had to face the difficulties of daily life. Mr Lin, a 61-year-old retired mid-level official said:

'... one dietitian came to explain how much meat I should eat, and how much fruit I should eat .... In reality, will we accept all that? Well, it's impossible!! ..., her calculations is right, ... but how can we, an ordinary family, prepare those things? ... The whole family cannot prepare and eat things to accommodate me every day ...'

The participants held diverse attitudes and practices. Some of them kept changing their behaviour over time and developed their own principles for food consumption, either giving in to practical limitations or modifying the restrictions. Miss Lai, a 44-year-old white-collar worker, expressing her views on diet after losing her left index finger in an accident:

'I may not necessarily die of diabetes (than from an accident). So, I might as well eat and enjoy (the food) here and now ...'

Mr Ho, also stressed:

'It'd be a lie if I said I didn't ever eat anything sweet .... We do our best to stick to today's quota ..., but if we drink it and it's sweet, then we shouldn't eat (any more sweets) for the rest of the day.'

\section{Exercise}

More than half of our participants (14/22) asserted that exercise does more good than harm. Some indicated that exercise could treat any existing disease and enhance physical strength if there was no disease (in Chinese: 'you bing zhi bing mei bing qiang shen'). Besides, we call 'activity' 'huo-dong' in Chinese, which literally means, 'survive-and-move'. So some participants said: 'if you want to survive, you have to keep moving'. Mr Chang talked to his parents-in-law:

'If you never exercise, your bodily functions will continue to degenerate ... You have to keep walking around; it's no good if you keep lying on your bed ... If you want to keep on living, you have to endure the pain, and keep moving ...'

Some participants indicated the benefit of exercise for reducing sugar level. Mr Chen argued:

'It's very reasonable, sugar helps us generating physical strength, and physical exercise consumes sugar, so the level (of sugar) goes down. That makes sense.'

Eliminating pharmaceutical toxins was considered as another important benefit, as Mr Chang's comment:

'There are hundreds of different types of drugs. There may be toxins inside, so we have to rely on exercise to (eliminate them through) sweating.' 
The 'sweating process' was linked to exercise frequently. Mrs Huang said:

'It is really comfortable to have a good sweat (in exercise) ...'

Some patients, inferring this linkage, argued that other activities producing sweating have the same effect as exercise (eliminating toxins or lowering blood sugar). Miss Pan, a $67-$ year-old housewife, said:

'Sometimes I didn't take (medication). Maybe the day after I took a spa or went bathing in a hot spring, I would stop taking my medication for a day. I did this many times. I just felt much better. I'm afraid it'll hurt my kidneys ...'

\section{Pharmaceutical treatment}

\section{Ambivalent responses}

Our participants held ambivalent attitudes towards pharmaceutical treatment. Some indicated the importance of medicines, and took it for granted. Mrs Huang and Mr Shu said:

'I think diabetic patients should listen to doctors, take drugs on time, ... and when you've run out of medicine, you have to go back to the hospital to get more.'

'If you've got diabetes, you have to take medicine, it's as simple as that ...'

But others worry very much about its potential adverse effects. Miss Chen said:

'I am not against taking medicine. It's just I'm afraid that there might be something wrong, you know, side-effects .... But I've to put my fears aside, I still take my medicine .... People often tell that too much medicine will damage kidneys .... I don't really know. It's bound to be bad if you take this (medicine) ...'

The most often-mentioned and mostly worried about longterm consequence of diabetes was renal injury (11/22), which was thought to be an adverse effect of hypoglycaemic agents. Only two mentioned heart disease or stroke as important complications. Mr Chang also had this comment:

'Some medication, well, I mean this type of medication is said to damage the kidneys if you take too much. I'm not sure if it is true. Is it? ...'

\section{Buffering the negative effect of pharmaceutical treatment}

In response to this ambivalence, some patients said they exercised to sweat (Mr Chang, described above), some immersed themselves in hot springs to sweat (Miss Pan, described above), Mr Chang and $\mathrm{Mr}$ Ho also talked about reducing drugs themselves:

'Some people take medicine for a while, quit a while, and then start again when there are fewer toxins left in your body. Or you have to quit while you are taking medicine for a common cold.'
'... if I don't eat anything sweet today, why should I take so many drugs? A few are quite enough. I would just take it in the morning and evening.'

Mr Chen, a 76-year-old retired teacher, has another measure: 'If I feel that I've taken a lot of drugs, I drink a lot of water ...'

\section{Discussion}

The findings of this study support the view that diabetic patients' explanations, perceptions and practices of illness management are interrelated as delineated in the models developed by Kleinman [15], Diefenbach and Leventhal [13] and Hampson [16]. Such inter-relationships were reflected in patients' self-adjustment which accomplish and integrate the three major strategies for diabetes management—drugs, exercise and diet control in daily life. For example, to avoid the believed 'toxicity' (especially renal toxicity) of hypoglycaemic agents, patients took more efforts in dietary restriction and physical exercise to decrease drug intake. Furthermore, some drank more water, did spa-bathing and physical exercise to sweat or 'pass water', and thus to 'eliminate drug toxins'. By doing so, patients can relieve dilemmas by combining diverse treatments. We detected three major beliefs and practices from the participants' statements. These culturally related beliefs and practices, though academically interesting, may have a negative influence on illness management.

First, all the rationale our participants held and the strategies they took were focused on 'sugar-control'. This misconception made some patients overlook the importance of managing other risk factors and some unnecessarily restricted their diet. As Chinese cuisine characterizes an important aspect of Chinese culture, a freedom to enjoy foods might play a critical role in their quality of life [40].

The most obvious reason why lay Chinese focus their attention on sugar-control is related to the name of diabetes ('Tang Niao Bing' in Chinese, translated from 'diabetes mellitus', means sugar-in-urine disorder). Most participants believed that diabetes is usually caused by over-consumption of sugar, so they focused their treatment on 'sugar control'. This notion is similar to Blumhagen's findings about 'hypertension', which was regarded by laypersons as 'Hyper-Tension' and characterized by excessive nervousness resulting from untoward social stress [41]. According to current medical knowledge, sugarcontrol is only one part of medical care. Body weight maintenance, blood pressure control, hypercholesterolaemia treatment and smoking cessation are clearly important in diabetes management [38]. Sucrose-containing foods even do not need to be restricted, and specialized diabetic diets are not necessarily superior to regular ones for the general population [36]. Using the term 'metabolic syndrome' [39] may be regarded as a specific kind of 'ti-zhi' ('constitution', a commonly accepted traditional term) [42] and reduce this problem of misconception and start a comprehensive management plan against diabetes. 
Second, in our participants' perceptions, exercise controls hyperglycaemia, prevents degeneration of the body and eliminates toxins via sweating. The first is compatible with medical knowledge and seems reasonable to laypersons, but the second and the third, with an origin in Chinese traditional medicine, may exercise unique impacts on patients' self-care behaviour. Most Chinese style exercises have different effects from those addressed by modern western medicine. For example, Kung$\mathrm{Fu}$ (also known as martial arts), is closely linked to the practice of traditional Chinese medicine [43] and is seen as a legitimate therapeutic modality [44]. Many Kung-Fu masters (Si-fu) are Chinese medicine doctors who often recommend health exercises, and particularly the soft form of martial arts, to their patients, while the hard form is suitable for sport and selfdefence [43]. Focusing on the therapeutic effectiveness of exercise may encourage diabetic patients to engage in physical activities, but the misconceptions of sweating and toxin elimination may have the opposite effect. One participant equated spa-bathing with exercise, and not only mistakenly thought that her exercise was enough, but also cut her pharmaceutical intake.

Third, our participants worried most that they were at high risk of 'renal injury' as a major adverse effect of hypoglycaemic agents. As a result, patients tried their best to reduce medication, without the knowledge that patients with strictly con- trolled blood sugar significantly reduce risk of microvascular complications (including nephropathy). This misconception may be accentuated by the metaphor of the kidney in Chinese culture. The kidney is seen as containing the inherited 'vital essence' and sperm. A kidney injury is viewed quite serious because of the tightly semantic network between lumbago, kidney deficiency (shen $\mathrm{xu}$ ) and loss of vital essence, which leads to shortened lifespan [30]. It is also difficult to persuade patients that hypoglycaemic agents are safe because most patients view western medicinal substance as artificial chemical compounds that are toxic, while herbal medicine, coming from natural sources, is safer and more compatible with human physiological functions [45].

In comparison with results of similar studies from different ethnicities [10,17,18,24-29] (Table 2), some of our findings are culture-specific, such as exercise's therapeutic/health promoting mechanism through meridian modulation, exercise's effect on eliminating pharmaceutical toxins and the worry of a pharmaceutically adverse effect of renal injury. Some were similar to that of other ethnic groups, including the strong conceptual linkage between consumption of sugary food and the onset of diabetes.

In conclusion, patients regard all treatment strategies as integrative and intertwined in daily life. The misconceptions of merely sugar control, sweating to eliminate drug toxins and

Table 2 Comparison of our findings with other studies and current orthodox medical recommendations

\begin{tabular}{|c|c|c|c|c|}
\hline & & & $\begin{array}{l}\text { Compared with other studies: C/S/ } \\
\mathrm{X} \dagger \text { (ref.) }\end{array}$ & Current orthodox medicine \\
\hline \multirow[t]{2}{*}{$\begin{array}{l}\text { Dietary } \\
\text { management }\end{array}$} & What they believe? & $\begin{array}{l}1 \text { Mainly cutting sugary food } \\
2 \text { Dietary management is beneficial/ } \\
\text { food is more compatible with natural } \\
\text { life than drugs }\end{array}$ & $\begin{array}{l}1 \text { (S) }[10,26] \text {, but may have unique } \\
\text { impact on Chinese daily life } \\
2 \text { (S) }[45]\end{array}$ & $\begin{array}{l}1 \text { Sugar control is only one } \\
\text { part of medical care; } \\
\text { restriction of sucrose } \\
\text { food is unnecessary }\end{array}$ \\
\hline & How they manage? & $\begin{array}{l}1 \text { Diverse attitudes and practices to } \\
\text { daily life limitations. Giving in/treat } \\
\text { with sophistication }\end{array}$ & $1(\mathrm{~S})[25]$ & \\
\hline Exercise & What they believe? & $\begin{array}{l}1 \text { More good than harm } \\
2 \text { Treat existing disease or improve } \\
\text { bodily strength if no disease. } \\
3 \text { Reduce sugar level } \\
4 \text { Eliminate pharmaceutical toxins } \\
5 \text { Emphasize benefits of sweating } \\
\text { process }\end{array}$ & $\begin{array}{ll}1 & (\mathrm{~S}) \text { [37] } \\
2 & \text { (C) originated from TCM } \\
3 & (\mathrm{~S})[37] \\
4 & (\mathrm{C})[29] \text { Vietnamese, also } \\
& \text { influenced by TCM } \\
5 & (\mathrm{~S})[10,29]\end{array}$ & $\begin{array}{l}1 \text { Compatible } \\
2 \text { Treating diabetes with } \\
\text { different mechanisms } \\
3 \text { Compatible } \\
4 \text { Not mentioned } \\
5 \text { Sweating } \neq \text { exercise }\end{array}$ \\
\hline \multirow[t]{2}{*}{$\begin{array}{l}\text { Pharmaceutical } \\
\text { treatment }\end{array}$} & What they believe? & $\begin{array}{l}1 \text { Ambivalence response: necessary } \\
\text { measures/worry about side-effects } \\
2 \text { Worry most about renal injury }\end{array}$ & $\begin{array}{l}1 \text { (S) Different cultures have different } \\
\text { origin of ambivalence }[29,47] \\
2 \text { (C) Exaggerated worries of renal } \\
\text { injury found in other ethnic } \\
\text { groups which are also influenced } \\
\text { by TCM [29] }\end{array}$ & $\begin{array}{l}1 \text { Hypoglycaemic agent } \\
\text { seems not to hurt the } \\
\text { kidney, but } \\
\text { hyperglycaemia does }\end{array}$ \\
\hline & How they manage? & $\begin{array}{l}\text { Buffering the negative effects: } \\
1 \text { exercise to sweat/ } \\
2 \text { immerse in hot spring or spa }\end{array}$ & $\begin{array}{l}1 \text { (X) [29] } \\
2 \text { (X) Also proposed by other clinical } \\
\quad \text { researchers [46] }\end{array}$ & \\
\hline
\end{tabular}

†C, Chinese culture specific finding, thought originated in traditional Chinese Medicine; S, shared finding with studies of other cultures; $\mathrm{X}$, uncertainty; $\ddagger$ TCM, traditional Chinese medicine. 
the more fundamental fear of renal toxicity of hypoglycaemic agents should be clarified during medical consultations. Renaming diabetes as 'metabolic syndrome' may bring new insights to Chinese diabetic patients.

\section{Acknowledgements}

This research was supported by Taichung Veterans' General Hospital research programme no. 915702A. We are also indebted to the public nurses for their help and the diabetic participants for giving us their own perspectives of living with diabetes. This research is also a part of the first author's (Dr Lai) PhD dissertation. We thank the members of the $\mathrm{PhD}$ dissertation advisory committee for their helpful comments in the design and implementation of the study.

\section{References}

1 Department of Health Taiwan (Republic of China). Health StatisticsVital Statistics. Taipei: Department of Health Taiwan, 2002.

2 World Health Organization. Global Burden of Diabetes. 1998. Available from: http://www.who.int/inf-pr-1998/en/pr98-63html (accessed 8 March 2003).

3 UK Prospective Diabetes Study (UKPDS) Group. Intensive bloodglucose control with sulphonylureas or insulin compared with conventional treatment and risk of complications in patients with type 2 diabetes (UKPDS 33). Lancet 1998; 352: 837-853.

4 Gaede P, Vedel P, Larsen N, Jensen GVH, Parving HH, Pedersen O. Multifactorial intervention and cardiovascular disease in patients with type 2 diabetes. N Engl J Med 2003; 348: 383-393.

5 Glasgow RE, Fisher EB, Anderson BJ, LaGreca A, Marrero D, Johnson SB et al. Behavioral science in diabetes: contributions and opportunities. Diabetes Care 1999; 22: 832-843.

6 Anderson RM, Robins LS. How Do We Know?: Reflections on qualitative research in diabetes. Diabetes Care 1998; 21: 1387-1388.

7 Wolpert H, Anderson BJ. Management of diabetes: are doctors framing the benefits from the wrong perspective? Br Med J 2001; 323: 994-996.

8 Bandura A. Self-efficacy: toward a unifying theory of behavioral change. Psychol Rev 1977; 84: 191-215.

9 Janz NK, Becker MH. The health belief model: a decade later. Health Education Q 1984; 11: 1-47.

10 Greenhalgh T, Helman C, Chowdhury AM. Health beliefs and folk models of diabetes in British Bangladeshis: a qualitative study. BMJ 1998; 316: 978-983.

11 Conner M, Norman P. The role of social cognition in health behaviours. In: Conner, M, Norman, P, eds. Predicting Health BehaviourResearch and Practice with Social Cognition Models. Buckingham: Open University Press, 1996: 1-22.

12 Schwarzer R, Reinbard F. Self efficacy and health behaviours. In: Conner, M, Norman, P, eds. Predicting Health Behaviour-Research and Practice with Social Cognition Models. Buckingham: Open University Press, 1996: 163-196.

13 Diefenbach MA, Leventhal $\mathrm{H}$. The common-sense model of illness representation: theoretical and practical considerations. J Social Distress Homeless 1996; 5: 11-38.

14 Good BJ. Illness representations in medical anthropology: a reading of the field. In: Medicine, Rationality, and Experience-an Anthropological Perspective. Cambridge: Cambridge University Press, 1994: 25-64.

15 Kleinman A. Patients and Healers in the Context of Culture. Berkeley: University of California Press, 1980.
16 Hampson SE, Glasgow RE, Toobert DJ. Personal models of diabetes and their relations to self-care activities. Health Psychol 1990; 9: 632-646.

17 Hampson SE, Glasgow RE, Foster LS. Personal models of diabetes among older adults: relationship to self-management and other variables. Diabetes Educator 1995; 21: 300-307.

18 Glasgow RE, Hampson SE, Strycker LA, Ruggiero L. Personal-model beliefs and social-environmental barriers related to diabetes selfmanagement. Diabetes Care 1997; 20: 556-561.

19 Freeman J, Loewe R. Barriers to communication about diabetes mellitus: patients' and physicians' different view of the disease. J Fam Pract 2000; 49: 507-512.

20 Rajwani R, Lipkin M Jr. The patient centered interview: research support. In: Lipkin, M Jr, Putnam, SM, Lazare, A, eds. The Medical Interview Clinical Care, Education, and Research. New York: Springer-Verlag, 1995: 530-537.

21 McWhinney IR. Clinical method. In: A Textbook of Family Medicine. New York: Oxford University Press, 1997: 129-178.

22 Susman JL, Helseth LD. Reducing the complications of type II diabetes: a patient-centered approach. Am Fam Physician 1997; 56: 471480.

23 Platt FW, Gaspar DL, Coulehan JL, Fox L, Adler AJ, Weston WW et al. 'Tell me about yourself': the patient-centered interview. Ann Intern Med 2001; 134: 1079-1085.

24 Cohen MZ, Tripp-Reimer T, Smith C, Sorofman B, Lively S. Explanatory models of diabetes: patient practitioner variation. Soc Sci Med 1994; 38: 59-66.

25 Murphy E, Kinmonth AL. No symptoms, no problem? Patients' understandings of non-insulin dependent diabetes. Family Prac 1995; 12: 184-92.

26 Schoenberg NE, Amey CH, Coward RT. Stories of meaning: lay perspectives on the origin and management of noninsulin dependent diabetes mellitus among older women in the United States. Soc Sci Med 1998; 47: 2113-2125.

27 Hunt LM, Valenzuela MA, Pugh JA. Porque me toco a mi? Mexican American diabetes patients' causal stories and their relationship to treatment behaviors. Soc Sci Med 1998; 46: 959-969.

28 Chesla CA, Skaff MM, Bartz RJ, Mullan JT, Fisher L. Differences in personal models among Latinos and European Americans: implications for clinical care. Diabetes Care 2000; 23: 1780-1785.

29 Mull DS, Nguyen N, Mull JD. Vietnamese diabetic patients and their physicians: what ethnography can teach us. Western J Med 2001; 175: 307-311.

30 Ots $\mathrm{T}$. The angry liver, the anxious heart and the melancholy spleen. The phenomenology of perceptions in Chinese culture. Cult Med Psychiatry 1990; 14: 21-58.

31 Krippner S. A cross-cultural comparison of four healing models. Altern Ther Health Med 1995; 1: 21-29.

32 Koo LC. The use of food to treat and prevent disease in Chinese culture. Soc Sci Med 1984; 18: 757-766.

33 Zhang J, Verhoef MJ. Illness management strategies among Chinese immigrants living with arthritis. Soc Sci Med 2002; 55: 1795-1802.

34 Jayne RL, Rankin SH. Application of Leventhal's self-regulation model to Chinese immigrants with type 2 diabetes. J Nurs Scholarsh 2001; 33: 53-59.

35 Miller WL, Crabtree BF. Primary care research: a multimethod typology and qualitative road map. In: Crabtree, BF, Miller, WL, eds. Doing Qualitative Research. California: Sage Publications, Inc., 1992: 3-30.

36 American Diabetes Association. Evidence-based nutrition principles and recommendations for the treatment and prevention of diabetes and related complications. Diabetes Care 2003; 25: 51S-61S.

37 American Diabetes Association. Physical activity/exercise and diabetes mellitus. Diabetes Care 2003; 26: 73S-77S.

38 American Diabetes Association. Standards of medical care for patients with diabetes mellitus. Diabetes Care 2003; 26: 33S-50S. 
39 National Cholesterol Education Program (NCEP). Expert Panel on Detection Evaluation and Treatment of High Blood Cholesterol in Adults. Third report of the National Cholesterol Education Program (NCEP) Expert Panel on Detection, Evaluation, and Treatment of High Blood Cholesterol in Adults (Adult Treatment Panel III) final report. Circulation 2002; 106: 3143-3421.

40 Yao G, Chung CW, Yu CF, Wang JD. Development and verification of validity and reliability of the WHOQOL-BREF Taiwan version. J Formos Med Assoc 2002; 101: 342-351.

41 Blumhagen D. Hyper-tension: a folk illness with a medical name. Cult Med Psychiatry 1980; 4: 197-224.

42 Lew-Ting CY, Hurwicz ML, Berkanovic E. Personal constitution and health status among Chinese elderly in Taipei and Los Angeles. Soc Sci Med 1998; 47: 821-830.

43 Koh TC. Chinese medicine and martial arts. Am J Chin Med 1981; 9: 181-186.

44 Cerrato PL. Tai chi: a martial art turns therapeutic. RN 1999; 62: 59-60.

45 Ernst E. Harmless herbs? A review of the recent literature. Am J Med 1998; 104: 170-178.

46 Hooper PL. Hot-tub therapy for Type 2 diabetes mellitus. N Engl J Med 1999; 341: 924-925.

47 Hunt LM, Valenzuela MA, Pugh JA. NIDDM patients' fears and hopes about insulin therapy: the basis of patient reluctance. Diabetes Care 1997; 20: 292-298. 